\title{
Characterizing low-activity waste containers: a case study for Compton Suppression Systems under challenging signal-to-noise ratio
}

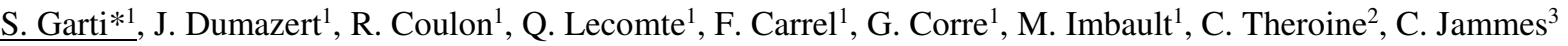 \\ ${ }^{1}$ CEA, LIST, Laboratoire Capteurs et Architectures Electroniques, 91191 Gif-sur-Yvette, France. \\ ${ }^{2}$ ORANO DS, Direction Technique et Innovation, 91191 Gif-sur-Yvette, France. \\ ${ }^{3}$ CEA, DEN, Laboratoire de Dosimétrie, Contrôle Commande et Instrumentation, 13108 S-Paul-lez-Durance, France. \\ *sara.garti@cea.fr
}

\section{Abstract}

This work investigates a compact Compton Suppression System (CSS) based on a High-Purity Germanium (HPGe) diode as a primary spectrometer. From a wide range of potential materials for the guard detector, including plastic scintillator, Thallium doped Sodium Iodide (NaI(Tl)), and Lanthanum III bromide $\left(\mathrm{LaBr}_{3}\right)$, a bismuth germanate crystal (BGO) is selected on account of a high photon mass-attenuation coefficient, thus allowing more compactness. The authors then describe the complete design study of an HPGe/BGO CSS based on the post-processing of Monte Carlo particle track history files (MCNP6.1/PTRAC). The calculation program, simulating the filtering of coincident events on both primary and secondary detectors, is validated through a series of experimental measurements using Am-241, Cs-137, and Co-60 sources in various configurations. On the basis of this empirically validated calculation, an optimization study of the BGO-active shield is performed over two factors of merit: Compton Suppression Factor $(C S F)$ and Full-Energy Peak Efficiency. Optimization steps subsequently involve the scaling of BGO internal and external radii, the thickness of the entrance window, and the diameter of a collimation hole. Eventually, performance figures of the CSS are illustrated in a case study involving the characterization of large waste containers, carrying low levels of uranium activity to seek among natural radiological background. Calculation results show a lowering of minimal detectable activities $(M D A)$ by a factor of 2.5 based on the signature of $1001-\mathrm{keV}$ gamma rays from $\mathrm{U}-238$.

\section{Introduction}

High-resolution gamma-ray spectrometry is a crucial tool for environmental monitoring, radiological safeguards, fundamental nuclear physics and astrophysics [1]. High-Purity Germanium (HPGe) crystals are most often selected as primary spectrometers due to their low bandgap $(0.67 \mathrm{eV}$ at $77 \mathrm{~K})$ coupled with a low Fano number equal to 0.13 , high atomic number $(\mathrm{Z}=32)$ and scalability [2]. Typical energy resolutions range between 1.7 and $2.3 \mathrm{keV}$ at the full-energy peak of $1.332 \mathrm{MeV}$ (Co-60) [3], leading to fine nuclide identification performance [4]. Spectrometers with dedicated front-end electronics and processing have been reported to operate above $10^{5}$ count per second $\left(\mathrm{c} \cdot \mathrm{s}^{-1}\right)$ input count rate (for instance [5], guaranteeing $3.2 \mathrm{keV}$ resolution at $1.332 \mathrm{MeV}$ under $3 \cdot 10^{5} \mathrm{c} \cdot \mathrm{s}^{-1}$ ), and up to the order of $10^{6} \mathrm{c} \cdot \mathrm{s}^{-1}$ in the case of the ADONIS analyser which maintain perfect life time measurement up to the Campbelling mode [6]. The combination of energy resolution, large-scale volume, and high impulse throughput ensures low minimum detectable activities (MDA) under a wide range of signal and noise magnitudes.

Quantitative spectrometry, allowing an estimation of nuclide activity, relies on the deconvolution of Full Energy Peaks (FEP). Such a deconvolution is complicated, especially in the low-energy range $(<200 \mathrm{keV})$ of the spectrum, because of the presence of Compton continuum. Although the intrinsic Peak-to-Compton ratio of the primary spectrometer ( $\mathrm{HPGe}$, in some cases $\mathrm{LaBr}_{3}$ ) may be high, there are measurement environments under which the impact of this continuum may be prohibitive. Two signal-to-noise ratio (SNR) configurations are paradigmatic of these impediments: 1) the need to detect low-activity nuclear fuel under average natural radiological background, as in the case of waste container characterization [7], wide-area decommissioning [8], environmental monitoring, and 2) in nuclear reactor environments [9] [10]. In both cases, the random fluctuation level of the Compton baseline with respect to the FEP signal degrades the detection limit of the spectrometry chain, thus preventing adequate and swift countermeasure.

Compton Suppression Systems (CSS) are designed and deployed to address nuclide identification and activity estimation under challenging SNR. In this paper, we describe the conception steps of an HPGe-based spectrometer including a CSS for the detection of down to $1 \mathrm{~Bq}^{-1} \mathrm{~g}^{-1}$ of natural uranium $(\mathrm{U})$ in $100-\mathrm{kg}$ Sorbalite $((\mathrm{Ca}(\mathrm{OH}) 2))$ waste containers. 


\section{State of the art}

Having orientated the material architecture of the gamma-ray detector towards an HPGe-CSS configuration, our first conception choice concerns the nature of the guard detector. After reviewing the principle of CSS, and establishing relevant figures of merit that will be used subsequently to assess the performance of any given design, we draw a state-of-the-art of CSS in the context of high-resolution gamma-ray spectrometry for low-activity characterization.

\section{A. Principle of anticoincidence Compton Suppression System}

$\mathrm{X}$-ray and gamma-ray attenuation involved two main processes: Compton and photoelectric effects. While photoelectric effects lead to FEP, Compton diffusion results in partial energy depositions, feeding spectral background as a continuum associated with a random noise. This continuum comes from (i) environmental background gamma rays, (ii) radioactivity within the diode assembly, (iii) radioactive impurities inside any shield around the spectrometer, and (iv) cosmic rays [11].

In $\gamma$-ray spectrometry, the detection of low intensity peaks is often complicated by the Compton continuum of higher energy $\gamma$-rays. This is the reason why Compton Suppression System have been introduced in the framework of low-noise measurement. In such systems, another detector, said "guard detector", is set around the primary spectrometer. Any couple of pulses recorded on the primary spectrometer and the guard sensor within a temporal correlation window is analysed as the signature of a partial energy deposition within the active shield, and thus rejected from the HPGe output spectrum as unable to contribute to FEP: such pulse processing is called anticoincidence filtering [12].

\section{B. Figures of Merit of CSS}

Related works use a variety of performance criteria to characterize CSS designs. The most commonly retained figures of merit (FoM) that account for both the FEP favouring and Compton continuum reduction are defined in references [13] and [14]. Based on these works, we hereby define one absolute and one relative FoM for our CSS assessment:

- Peak-to-Compton ratio, labelled $\frac{P}{C}$ : this is the ratio of the number of counts under a FEP of interested (defined between the $m^{\text {th }}$ and the $n^{\text {th }}$ channel), to the number of counts over a spectral range corresponding to the Compton region (between the $k^{\text {th }}$ and the $j^{\text {th }}$ channel) left to the FEP. If we have $N_{i}$ the number of counts recorded inside the $i^{\text {th }}$ channel, then the $\frac{P}{C}$ FoM reads

$$
\frac{P}{C}=\frac{\frac{1}{n-m+1} \sum_{i=m}^{n} N_{i}}{\frac{1}{j-k+1} \sum_{i=k}^{j} N_{i}}
$$

- Compton Suppression Factor for $\frac{P}{C}$, labelled $C S F_{P / C}$ : this is the ratio of the anticoincidence filtered Peak-toCompton ratio to that of unfiltered Peak-to-Compton ratio, i.e.

$$
C S F_{P / C}=\frac{\left(\frac{P}{C}\right)_{\text {filtered }}}{\left(\frac{P}{C}\right)_{\text {unfiltered }}}
$$

According to the authors, the Compton continuum lower bound will take values between 0 and the backscattering peak, while the upper band will lie between the Compton edge and the last energy bin before FEP. As for the radionuclides selected for the assessment of Compton reduction, the most common candidates are Cs-137 (single gamma ray of energy $661.7 \mathrm{keV}$ ) and Co-60 (two coincident gamma rays of energies $1173.2 \mathrm{keV}$ and $1332.5 \mathrm{keV}$ ) [15]. Regarding this procedure, we should point out that CSS suppress the coincident signature of cascade emissions (such as the ones of $\mathrm{Co}-60$ and $\mathrm{Na}-24$ ), so that a fraction of physically-relevant, high-energy summations will be lost in presence of radio-emitters with a multiplicity higher than 1 . 


\section{Material for the guard detector}

A literature search was carried out in order to characterize the major design options of the Compton Suppression System (nature and dimensions of guard detector, SF...). A summary of CSS reported in literature is given in Table I.

Table I. Characteristics of CSS in reference work

\begin{tabular}{|c|c|c|c|c|c|c|c|c|}
\hline \multicolumn{2}{|c|}{ Primary Spectrometer (PS) } & \multicolumn{3}{|c|}{ Guard Detector (GD) } & \multicolumn{3}{|c|}{ Suppression Factor } & \multirow[b]{2}{*}{ References } \\
\hline Type & $\begin{array}{c}\text { Relative } \\
\text { Efficiency }(\%)\end{array}$ & Type & $\begin{array}{l}\text { Diameter } \\
(\mathrm{mm})\end{array}$ & $\begin{array}{c}\text { Length } \\
(\mathrm{mm})\end{array}$ & Cs-137 & Co-60 & $\mathrm{Na}-24$ & \\
\hline BEGe & & $\mathrm{NaI}$ & & & 4.28 & 2.84 & & [13] \\
\hline BEGe & & BGO & & & 4.71 & 8.36 & & [13] \\
\hline HPGe & 10,8 & $\mathrm{NaI}$ & 25,4 & 30.6 & 12.6 & 32 & & [16] \\
\hline HPGe & 25 & $\mathrm{NaI}$ & 22,9 & 25.4 & 5.2 & & 7 & {$[17]$} \\
\hline HPGe & 24 & BGO & 16,4 & 27.6 & & 39.5 & & [18] \\
\hline HPGe & $20-25 \%$ & BGO & 12,7 & 15.2 & & 4 & & [19] \\
\hline $\mathrm{HPGe}$ & 20 & $\mathrm{NaI}$ & 30 & 35 & & 7.2 & & [20] \\
\hline $\mathrm{Ge}$ & 20 & $\mathrm{NaI}$ & 30 & 35 & & 7.4 & & [21] \\
\hline $\mathrm{Ge}$ & 25 & BGO/NaI & & & & 11 & & [22] \\
\hline
\end{tabular}

As reflected in Table I, thallium doped Sodium Iodide ( $\mathrm{NaI}(\mathrm{Tl})$ ) and bismuth germanate crystal (BGO) detectors are the most commonly used active shields in HPGe-based CSS [13],[16-22]. These veto materials give access to Suppression Factor $S F$ ranging from 2.5 to 39.5, depending on geometrical design, radionuclide and definition of the Compton region. The selection of a type of guard detector will be based on a series of physical properties, whose main features are listed in Table II for BGO, $\mathrm{NaI}(\mathrm{Tl})$, cerium-doped lanthanum Bromide III ( $\mathrm{LaBr}_{3}(\mathrm{Ce})$ ) and polyvinyltoluene (PVT) plastic scintillators.

Table II. Physical properties of guard detectors for CSS

\begin{tabular}{|c|c|c|c|c|}
\hline Physical property & $\mathrm{NaI}(\mathrm{Tl})$ & BGO & $\mathrm{LaBr}_{3}$ & Plastic \\
\hline Density $\left(\mathrm{g} . \mathrm{cm}^{-3}\right)$ & 3.67 & 7.13 & 5.06 & 1.032 \\
\hline Light yield (photons.keV ${ }^{-1}$ ) & 38 & 8 & 63 & 10 \\
\hline Decay time (ns) & 250 & 300 & 25 & 2 \\
\hline Relative energy resolution (@662 keV) & $7 \%$ & $9 \%$ & $3 \%$ & - \\
\hline
\end{tabular}

Compton suppression relies on the detection of a maximum number of scattered photons inside the active shield. Now, the linear attenuation coefficient from Compton scattering ( $\left.\mu_{\text {Compton }}\right)$ varies linearly with density, thus orientating our choice toward high-density scintillators. Moreover, CSS for field applications have to be as compact as possible, which tends to exclude plastic scintillators due to their low density: the same attenuation as BGO would, for instance, require seven times the same range in PVT. BGO also has higher density than $\mathrm{LaBr}_{3}$ and NaI: consequently, the $95-\%$-attenuation of $1-\mathrm{MeV}$ photon beam would require up to $145 \mathrm{~mm}$ of $\mathrm{NaI}$ or $100 \mathrm{~mm}$ of $\mathrm{LaBr}_{3}$, against $65 \mathrm{~mm}$ of BGO [13]. Nevertheless, $\mathrm{LaBr}_{3}(\mathrm{Ce})$ scintillator exhibits a lower decay time than BGO (or $\mathrm{NaI}(\mathrm{Tl})$ ), enabling them to perform with less pile-up under higher input count rates. Though this factor of merit could be useful in the case of high-activity monitoring, it is less relevant for the type of characterization we are investigating in this paper, where the isotopic activity of interest (around a total of $100 \mathrm{kBq}$ to be detected in one hour) is of the same order of environment background. Similarly, better energy resolutions achievable with $\mathrm{LaBr}_{3}$ and $\mathrm{NaI}$ are only valuable when the guard detector is additionally used as a spectrometer, which is not the case in the CSS architecture we consider. It follows that BGO was selected as the best candidate for the application at hand.

\section{Passive shield}

In many fixed spectrometric benches, environmental background is reduced by shielding both the primary spectrometer and the surrounding guard detector with a thickness of dense passive shield, typically $10-15 \mathrm{~cm}$ of lead $(\mathrm{Pb})$. As an illustration, the addition of a $10-\mathrm{cm} \mathrm{Pb}$-shield allowed a background reduction by a factor of 50 in the $\mathrm{NaI}(\mathrm{Tl})$-guard detector in reference [23]. In addition, lead shields are often covered with a few millimetres of copper to attenuate $75-80 \mathrm{keV} \mathrm{X}$ rays from $\mathrm{Pb}$, followed by Lucite [9] or, preferably, with an intermediate layer of tin in-between [24]. 


\section{Numerical model of the HPGe/BGO CSS}

After selecting the types of spectrometer and shield, we present below the next step to design an experimentally validated tool for modelling filtered and unfiltered responses of the HPGe/BGO CSS. In this section, we describe the building and numerical pre-validation of a MNCP-based Compton Suppressor model.

\section{A. Material building blocks of the model}

The model of the primary spectrometer is based on an HPGe detector commercialized by Mirion Technologies CANBERRA, and referenced as REGe1020 [25]. Data from the manufacturer are combined with an X-ray radiography of the diode (Fig. 1a)) so the layout of every component may be determined. In the model, the Ge crystal cylinder is $4.64(\mathrm{H}) \times 4.62(\varnothing) \mathrm{mm}$, the beryllium window is $0.5-\mathrm{mm}$ thick. The aluminium end cap, the copper cold finger, dead layers and vacuum spaces within the detector are also modelled. The accurate dimensions of the diode have been tuned by a patented technique based on simulated reheat technique ensuring the optimisation of the 22 parameters of the model using experimental points obtained by a ${ }^{152}$ Eu reference source [26].

The primary spectrometer is surrounded by a single BGO-annulus with crystal thickness of $25 \mathrm{~mm}$ and $100 \mathrm{~mm}$ height, mounted in a 3-mm aluminium housing. An 11.5-cm Pb-shield, containing both the primary and the guard detectors, is added to the simulation in view of the experimental calibration. The geometry is represented in Fig. 1b) and $\mathrm{c}$ ).
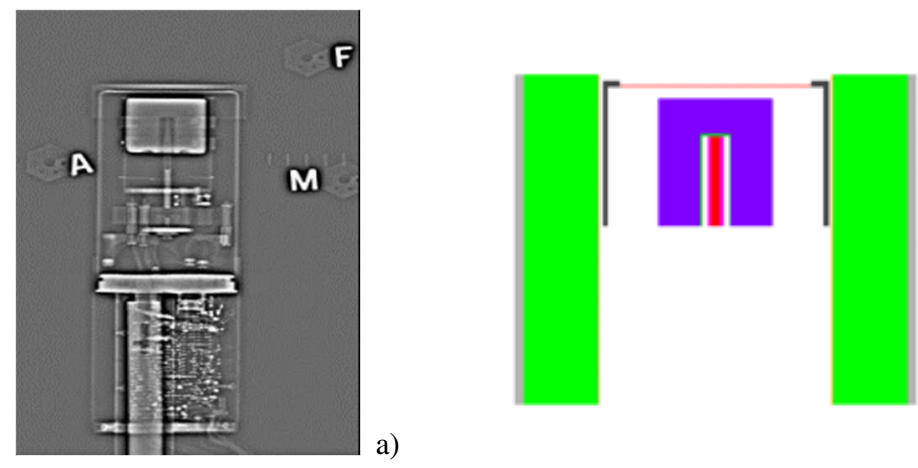

b)

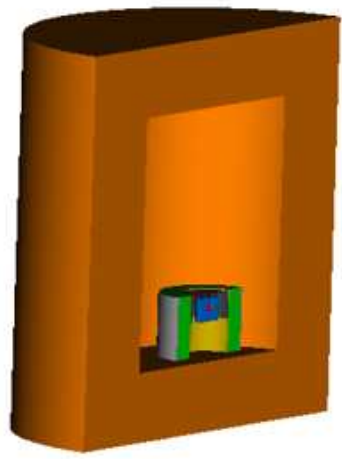

c)

Fig. 1 a) X-ray radiography of REGe1020, b) Visualization (Moritz 1.23 [27]) of MCNP6.1 material model, 3-D cross-section view of CSS cross-section view of spectrometer and active shield (HPGe detector is shown in blue, the cold finger is shown in red, and the BGO is shown in green, aluminium housing in black for HPGe and grey for BGO), and c) 3-D cross-section view of CSS inside the $\mathrm{Pb}$-shield for experimental calibration.

\section{B. Building of a numerical code for the study of gamma-ray anticoincidence filtering}

MCNP6.1 is a software package developed by Los Alamos National Laboratory, relying on Monte Carlo method to simulate the transport of particles in matter [28]. In this study, the output quantity of interest is given by the pulse height tally of MCNP (response function 8), corresponding to a spectral deposition yield labelled $\tau_{M C N P}(E)$ and expressed in energy depositions per generated source particle (in our case, gamma rays) and per energy bin (d. $\gamma^{-1}$ ). Throughout the entire numerical study, we used single- or multi-energy (for instance, $661.7 \mathrm{keV}$ with $84.9 \%$ line intensity for Cs-137, as well as X rays from internal conversions between $31 \mathrm{keV}$ and $37 \mathrm{keV}$ ) source terms. The comparative use of other particle transport packages, such as GEANT4 with libraries dedicated to given nuclides and physical processes associated to their decay [29], was left as an outlook in the course of this work. Moreover, to account for the experimentally observed resolution of the spectrum, a Gaussian Energy Broadening (GEB) card is added to Monte Carlo simulations [30]. A Gaussian broadening around a FEP at energy $E$ is characterized by its Full Width at Half Maximum $F W H M(E)$. The latter is specified in GEB card by means of two parameters $a$ and $b$ so that

$$
F W H M(E)=a+b \sqrt{E}
$$

Parameter $a$ relates to electronic noise, while $b$ depends on the number of charge carriers created inside the semiconductor and possible correlations [31]. The value for these parameters were determined by carrying an energy resolution calibration of the REGe1020 detector by means of a multiple-gamma-ray Eu-152 source. The least-meansquare adjustment of experimental FHWM values gave values $\left\{a=0.025 \mathrm{MeV} ; b=1.84 \mathrm{MeV}^{-1 / 2}\right\}$. In order to 
208 perform a quantitative study of the simulation scheme, the energy deposition yield $T_{M N C P}(E)$ at a FEP of energy $E$ 209 will therefore be estimated as

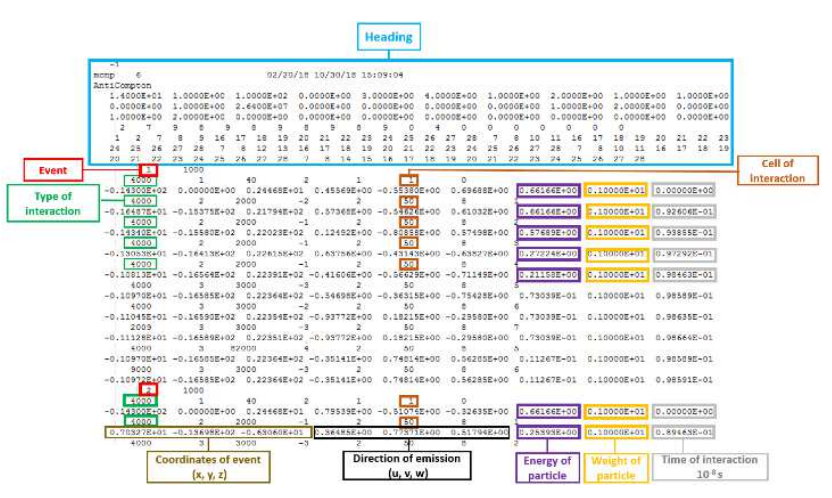

228

$$
T_{M N C P}(E)=\sum_{E_{i} \in[E-1.25 \cdot F W H M(E) ; E+1.25 \cdot F W H M(E)]} \tau_{M C N P}\left(E_{i}\right)
$$

While the numerical scheme described above is sufficient to obtain simulation-based estimates of $P_{\text {unfiltered }}$ and $C_{\text {unfiltered }}$ as used in Eq. (1), (2) and (5), it will not provide us estimates of $P_{\text {filtered }}$ and $C_{\text {filtered }}$ after anticoincidence filtering. Indeed, the pulse height tally only provides a macroscopic estimate of a physical quantity, in this case the sensor response, computed over a large number of random draws. It does not give access, however, to the complete history of individual particles, which is necessary to identify detection events that occur quasisimultaneously in both detectors and will be rejected by the anticoincidence filter. Particle TRAck files (PTRAC) output files contain the full history of a particle interactions within matter from its generation to its disappearance. Fig. 2a) reproduces the content of such a PTRAC file. A code with Matlab R2017b [32] was thus developed to read and extract automatically useful information from the PTRAC files, including the type of interaction undergone by the particle, the number of the cell in which the interaction took place, the involved nuclide, and energy of the particle before and after the interaction.

Event-by-event post-processing and reconstruction of filtered spectra are carried out following the flow chart depicted in Fig. 2b). The energy deposited in the primary spectrometer "PS" and the guard detector "GD" is determined at every step, and binned to appropriately reconstruct the expected spectrum $\tau_{P T R A C \text {,unfiltered }}(E)$ before filtering.

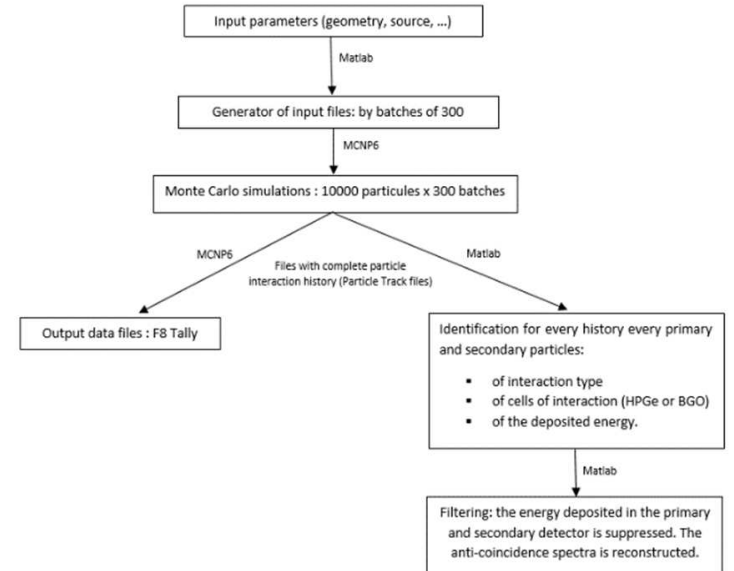

Fig. 2a) MCNP6.1 Particle TRAck file, and b) Flow chart of Particle TRAck file post-processing.

For a given history " $i$ ", if we label $E_{i, 1}$ and $E_{i, 2}$ the energies laid down in PS and GD respectively, the anticoincidence function that produces the filtered response $\mathrm{E}_{\mathrm{i}, 3}$ in PS reads

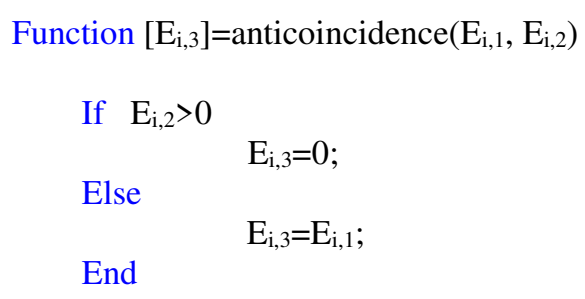

\section{End}

We note that, if we do not take apply the anticoincidence filtering function, the spectrum of the PS reconstructed 247 MCNP6.1. Based on this argument, we performed a first numerical validation study, as detailed in the next paragraph. 248 We also point out that we did not make use of the temporal information associated to interactions events in PTRAC 249 files. Time-based rejection conditions, implementing the experimental gate delay in the anticoincidence filtering, 250 would lead to lower the estimated number of Compton suppressed events. However, the said gate is not adjusted with 251 respect to the kinematics of radiation-matter interaction (the average delay between physically correlated, consecutive 
events we are filtering being in the order of $10 \mathrm{~ns}$ ), but according to the time properties of the semiconductor and scintillator pulses at the output of the processing chain. This explains why the temporal width of the filter would be in the order of $1 \mu \mathrm{s}$. Such a delay will allow fortuitously coincident gamma-ray events to be mistakenly filtered as correlated energy depositions. Now, as source particles in MCNP6.1 trials are generated independently, and the time "shakes" reported in PTRAC files are reinitialized for every new trial, it is not directly possible to account for this effect in the post-processing scheme we are introducing. As a result, we chose, in this first version, not to account for half of the distortions induced by time considerations in the model, but rather build the simulator from an "ideal" filtering of physically correlated events, and calibrated such a model with experimental data capturing the consequences of both phenomena, as it will described in throughout Section IV. Refinements of the numerical scheme addressing the influence of the anticoincidence gate, as well as pile-up, have thus been left as outlooks of this work.

\section{Pre-validation study of anticoincidence filtering simulation}

In order to pre-validate the numerical processing simulator described in Section III.B, we compared the reconstructed spectrum $\tau_{P T R A C \text {, unfiltered }}(E)$ obtained without implementing any anticoincidence filtering to the output $\tau_{M C N P}(E)$ of tally 8 (pulse height tally of MCNP6.1). Both spectral responses to a Cs-137 emission source term are superimposed in Fig. 3, where the agreement can qualitatively observed.

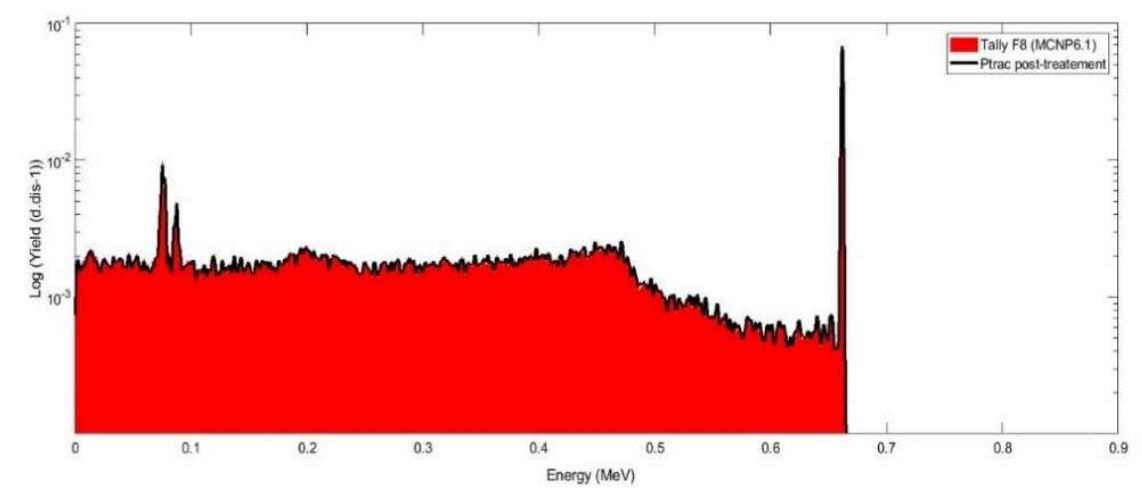

Fig. 3. Simulated spectral yields $\tau_{M C N P}(E)$ and $\tau_{P T R A C, \text { unfiltered }}(E)$ (Cs-137 gamma-ray source term).

A quantitative comparison is drawn by computing the relative difference $\varepsilon_{\%}(E)$ between both simulated spectral responses $T_{M N C P}(E)$ and $T_{P T R A C \text {, unfiltered }}(E)$, integrated around an energy of interest $E$ for the radionuclide. The summation intervals are defined as shown in Eq. (7) when $E$ is the centroid of a FEP, and correspond to a relevant spectral window $[E-\Delta ; E+\Delta]$ otherwise:

$$
\varepsilon_{\%}(E)=100 \% \cdot \frac{T_{P T R A C, \text { unfiltered }}(E)-T_{M C N P}(E)}{T_{M C N P}(E)}
$$

$$
\varepsilon_{\%}(E)=100 \% \cdot \frac{\sum_{E_{i} \in[E-\Delta ; E+\Delta]} \tau_{P T R A C, \text { unfiltered }}\left(E_{i}\right)-\sum_{E_{i} \in[E-\Delta ; E+\Delta]} \tau_{M C N P}\left(E_{i}\right)}{\sum_{E_{i} \in[E-\Delta ; E+\Delta]} \tau_{M C N P}\left(E_{i}\right)}
$$

The values of $\varepsilon_{\%}(E)$ that are shown in Table III correspond to four regions of interest for Cs-137: FEP at $661.7 \mathrm{keV}$, Compton edge at $477.4 \mathrm{keV}$, backscatter peak at $184.3 \mathrm{keV}$, and the middle of the Compton plateau around $331 \mathrm{keV}$.

Table III. Relative discrepancies between MCNP6.1 tally and PTRAC post-treatment for Cs-137

\begin{tabular}{ccccc}
\hline Spectral region & $\begin{array}{c}\text { Backscatter Peak } \\
(\mathbf{1 8 4} \pm \mathbf{2 0} \mathbf{~ k e V})\end{array}$ & $\begin{array}{c}\text { Compton Plateau } \\
(\mathbf{3 3 1} \pm \mathbf{2 0} \mathbf{k e V})\end{array}$ & $\begin{array}{c}\text { Compton Edge } \\
(\mathbf{4 7 7} \pm \mathbf{2 0} \mathbf{k e V})\end{array}$ & $\begin{array}{c}\text { FEP } \\
(\mathbf{6 6 1 . 7} \pm \mathbf{3} \mathbf{~ k e V})\end{array}$ \\
\hline$\varepsilon_{\%}$ & $-1.6 \pm 0.1 \%$ & $2.6 \pm 0.1 \%$ & $2.5 \pm 0.1 \%$ & $3.7 \pm 0.2 \%$ \\
\hline
\end{tabular}

Calculations give relative discrepancies $\varepsilon_{\%}(E)$ below $5 \%$ in modulus, hence providing a first validation of the accuracy of the numerical scheme constructed for this study, and described in paragraph III.B. We will therefore rely on this simulator to compute the expected spectral response $\tau_{P T R A C \text {,filtered }}(E)$ when implementing a Compton anticoincidence suppression. 


\section{Estimated FoM of CSS standard configuration}

Having built a numerical simulator of the standard configuration of the HPGe/BGO CSS depicted in Fig. 2b), we investigated the expected spectral responses of the PS, first without any active shield, and second, with the addition of the GD, with and without implementing anticoincidence filtering. Three configurations were thus simulated, that are indexed $\left\{s_{1}, s_{2}, s_{3}\right\}$ as follows:

- $\quad \mathrm{s}_{1}$ : HPGe only in Pb-shield;

- $\quad \mathrm{s}_{2}$ : HPGe and BGO integrated in Pb-shield without anticoincidence filtering;

- $\quad \mathrm{s}_{3}$ : HPGe and BGO integrated in Pb-shield with anticoincidence filtering.

Comparison between configurations $s_{1}$ and $s_{2}$ enables us to evaluate the impact of the passive influence of the GD on the PS response, as BGO is a dense and high-atomic-number material. By comparing $s_{2}$ and $s_{3}$, we quantify the performance of CSF with respect to Compton reduction. All three configurations were simulated with Am-241, Cs137, and Co-60 source terms. Additionally, four axial positions P1 to P4 were studied for the point-like source, as defined in Fig. 4a). Compton suppression is illustrated in Fig. 4b) with a Cs-137 source term, over the entire range between the first channel and the 661.7-keV FEP. We notice especially the reduction of X-ray peaks in the lower energy region. Those of $\mathrm{Pb}$ fluorescence peaks at $74.8 \mathrm{keV}$ and $85.7 \mathrm{keV}$ from the passive shield, potentially superimposed with Bi fluorescence peaks at $77.1 \mathrm{keV}$ and $87.3 \mathrm{keV}$ from the BGO guard detector [33].

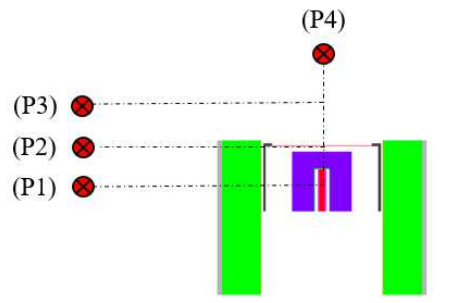

314

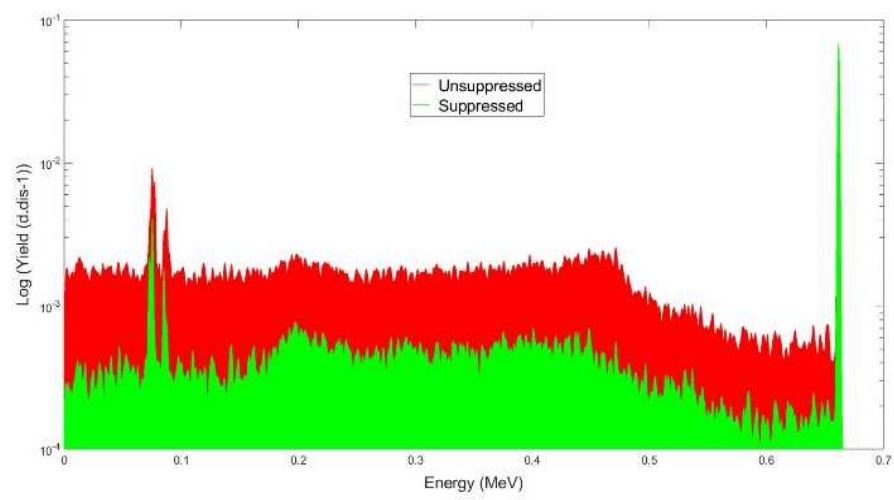

Fig. 3a) Simulated irradiation positions, and b) unfiltered ( $\left.s_{2}\right)$ and filtered $\left(s_{3}\right)$ spectra with HPGe/BGO.

The expected performance of the standard CSS design hereby modeled is assessed with the FoM $C S F_{P / C}$ as defined in Eq. (2). Indeed and by construction, $s_{2}$ gives access to a numerical estimate of $\left(\frac{P}{C}\right)_{\text {unfiltered }}$, while $s_{3}$ allows us to compute an expected value for $\left(\frac{P}{C}\right)_{\text {filtered }}$. The results of this simulation study are summarized in Table IV, as function of radionuclide emission spectrum and position of the source.

Table IV. Compton Suppression Factors $C S F_{P / C}$ with HPGe/BGO in standard configurations

\begin{tabular}{cccccc}
\hline Nuclide & Energy (keV) & CSF (P1) & CSF (P2) & CSF (P3) & CSF (P4) \\
\hline Am-241 & 59.5 & 1.0 & 1.0 & 1.0 & 1.0 \\
Cs-137 & 661.7 & 3.8 & 3.7 & 2.8 & 2.8 \\
Co-60 & 1173.2 & 3.6 & 3.5 & 3.0 & 2.6 \\
Co-60 & 1332.5 & 3.6 & 3.6 & 3.1 & 2.6 \\
\hline
\end{tabular}

For medium or high-energy source terms, such as the ones of Cs-137 and Co-60, the values of $C S F_{P / C}$ over all four positions lie between 2.6 and 3.8, with an average of 3.2 and a one-sigma standard deviation of 0.4 . We observe that that $C S F_{P / C}$ decreases of about 1 when rotating from $\mathrm{P} 1$ to $\mathrm{P} 4$, which is straightforwardly explained by the fact that incident gamma rays then have a shorter course within the GD, and thus undergo less Compton diffusions that that can be filtered with anticoincidence. On the contrary, due to the strong attenuation of low-energy photons by BGO, the implementation of the filter has no influence on the metrological FoM of the CSS for simulated Am-241. 
The numerical results reported in Table IV are representative of the work of an "ideal" multiple-interaction filter, leading to the 100-\% rejection of incoming photons that scatter inside the GD and 100-\% conservation of photons that are fully absorbed inside the PS. The actual performance of active shielding will depend on the pulse-processing analog and digital architecture, dead time, and the boundaries of the temporal windows for coincident event filtering [13]. The next step of our study is then to evaluate, through a series of measurements, the experimental calibration coefficient of the CSS numerical model described in this section.

\section{Experimental calibration of HPGe/BGO CSS model}

\section{A. Experimental setup}

The material buildings blocks of the test bench were described in paragraph III.A. The setup, as reproduced in Fig. 4a), is based on a Reverse-Electrode high-purity germanium REGe1020 from Mirion Technologies CANBERRA [25], with a 10-\% relative efficiency and FWHM of $1 \mathrm{keV}$ and $2 \mathrm{keV}$ respectively at $122 \mathrm{keV}$ and $1332 \mathrm{keV}$. The single-annulus guard detector was obtained from Scionix Holland [34]: it contains is 25-mm-thick and 100-mm-high BGO crystal, mounted in a 3-mm-thick aluminium housing including four 25.4-mm-diameter R1924A Photomultiplier Tubes (PMTs) from Hamamatsu. The PMTs are biased in high voltage with a CAEN N147 fourchannel programmable power supply. The BGO detector shows a $16.3-\%$ a resolution at $661.7 \mathrm{keV}$. The HPGe/BGO CSS is mounted in a 11.5 -cm-thick cylindrical $\mathrm{Pb}$ castle, providing a complete $360-^{\circ}$ shielding against external background radiation.

The acquisition and treatment of both the Ge semiconductor and the BGO scintillator channels are performed by means of CAEN "Hexagon" MCA and pulse processor [35]. The ADC digitizes the pulses with a 100-MHz sampling frequency, while processing involves trapezoidal filtering and differentiation of the signal. The pulses are extracted using a fast trigger, and maximum amplitudes recorded in a 16-bit height histogram.

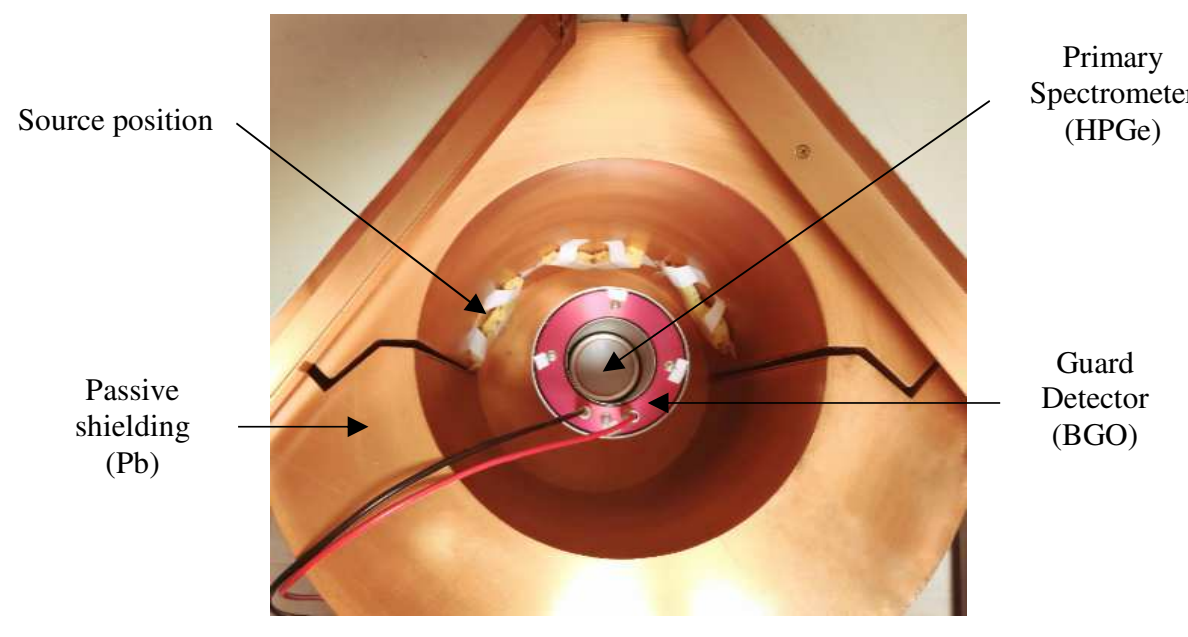

Fig. 4 Test bench for the HPGe/BGO setup, the main detector is set at the center. It is surrounded by a cylindrical BGO (in red). For the sake of better visualisation, only positions $\mathrm{P} 1, \mathrm{P} 2$ and $\mathrm{P} 3$ are displayed.

The temporal width of the anticoincidence filter on the PS and GD channels is adjusted via CAEN MC ${ }^{2}$ Analyzer software. As discussed in the end of Section III, the setting of the coincidence rejection window impacts the overall spectrometric performance of the CSS. Indeed, as the gate widens, the risk of rejecting unrelated events that are truly coincident within the bounds of the window increases (increase of fortuitous coincidences), to the resulting detriment of the FEP statistic. Conversely, as the gate gets narrower, a smaller number of true coincidence events will be filtered, to the detriment of Compton baseline reduction strength. Therefore, the definition of the anticoincidence window results from a trade-off, whose optimum was experimentally observed around $1.5 \mu \mathrm{s}$.

\section{B. Calibration of CSS model without anticoincidence filtering}

The three irradiation configurations $\left\{\mathrm{s}_{1}, \mathrm{~s}_{2}, \mathrm{~s}_{3}\right\}$ detailed in paragraph III.D have been experimentally reproduced in the four positions $\{\mathrm{P} 1, \mathrm{P} 2, \mathrm{P} 3, \mathrm{P} 4\}$. For each measurement configuration and positions, three reference sources have been used: Cs-137 (activity $A=500 \mathrm{kBq})$, Co-60 (A=110 kBq), and Am-241 (A=3.97 MBq). The experimental FEP response is labelled $R_{\text {unfiltered }}(E)$ and expressed in count per second $\left(\mathrm{c} . \mathrm{s}^{-1}\right)$. To compare homogenous quantities, the simulated spectral yield at the FEP $T_{P T R A C, \text { unfiltered }}(E)$, in d. $Y^{-1}$, must be converted into a simulated spectral response, labelled $Y_{\text {unfiltered }}(E)$ and expressed in ${\mathrm{d} . \mathrm{s}^{-1}}^{-1}$ using the equation: 

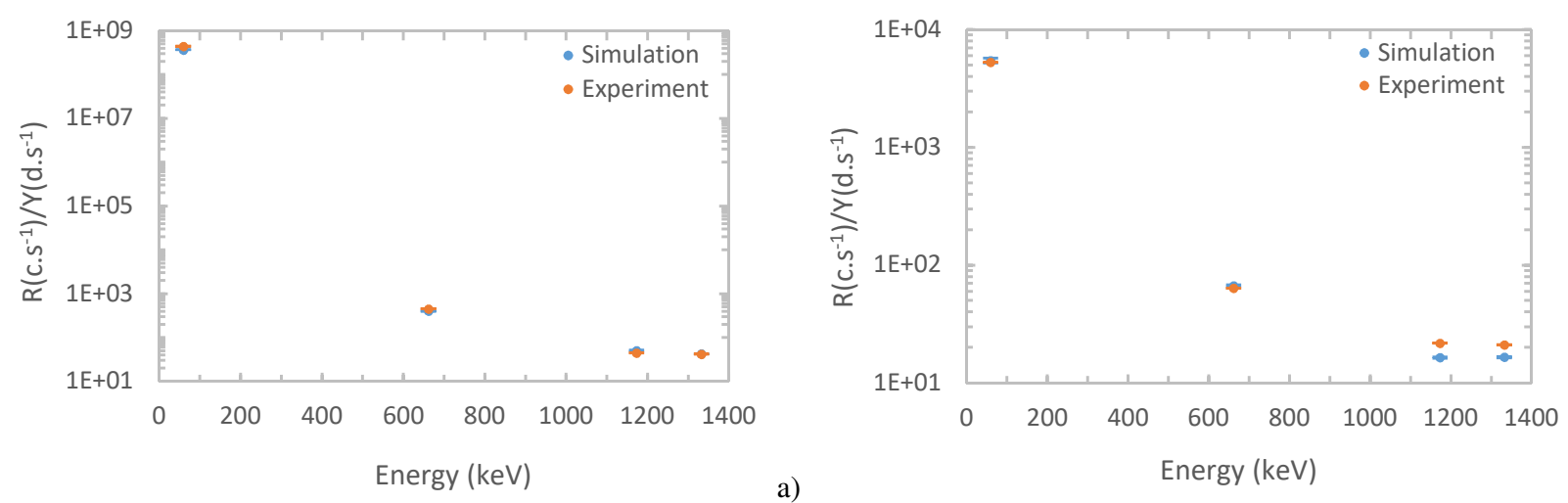

With $I_{\gamma}$ the intensity factor of the underlying gamma ray. Figure 5 presents the experimental $R_{\text {unfiltered }}(E)$ and simulated $Y_{\text {unfiltered }}(E)$ responses in configurations $\mathrm{s}_{1}$ and $\mathrm{s}_{2}$ at $\mathrm{P} 2$ position.

Fig. 5 a) Simulated and measured responses in $s_{1}$ configuration at $\mathrm{P} 2$ position b) Simulated and measured responses in $s_{2}$ configuration at $\mathrm{P} 2$ position.

Figures 5a) and b) show that $R_{\text {unfiltered }}(E)$ and $Y_{\text {unfiltered }}(E)$ have similar trends for all energies of interest. It can be noted that the sole presence of BGO, without any anticoincidence filtering, induces a loss of count rates by

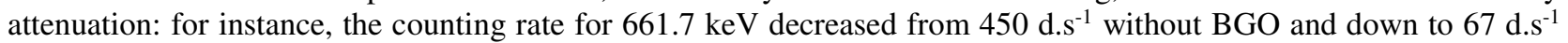
with BGO.

If we label $(E, P)$ an energy-position couple, we can estimate the value of an experimental calibration factor $\omega(E, P)$ of our simulation model such as 416 calculated from $\tau_{P T R A C \text {, filtered }}(E, P)$ the same way as in Eq. (9). The corresponding experimental responses $417 R_{\text {unfiltered }}(E, P)$ and $R_{\text {filtered }}(E, P)$ are obtained by means of the electronic chain described in paragraph IV.A. As a 418 figure of merit for our model, we directly use the simulation-based and experimentally-obtained values of Compton 419 Suppression Factor, labelled $C S F_{\text {exp }}$ and $C S F_{\text {sim }}$. The values of $C S F_{\text {exp }}$ and $C S F_{\text {sim }}$ are plotted in Fig. 6a) at position $420 \mathrm{P} 1$ as a function of energy, and in Fig. 6b) at energy $1.17 \mathrm{MeV}$ as a function of position. 

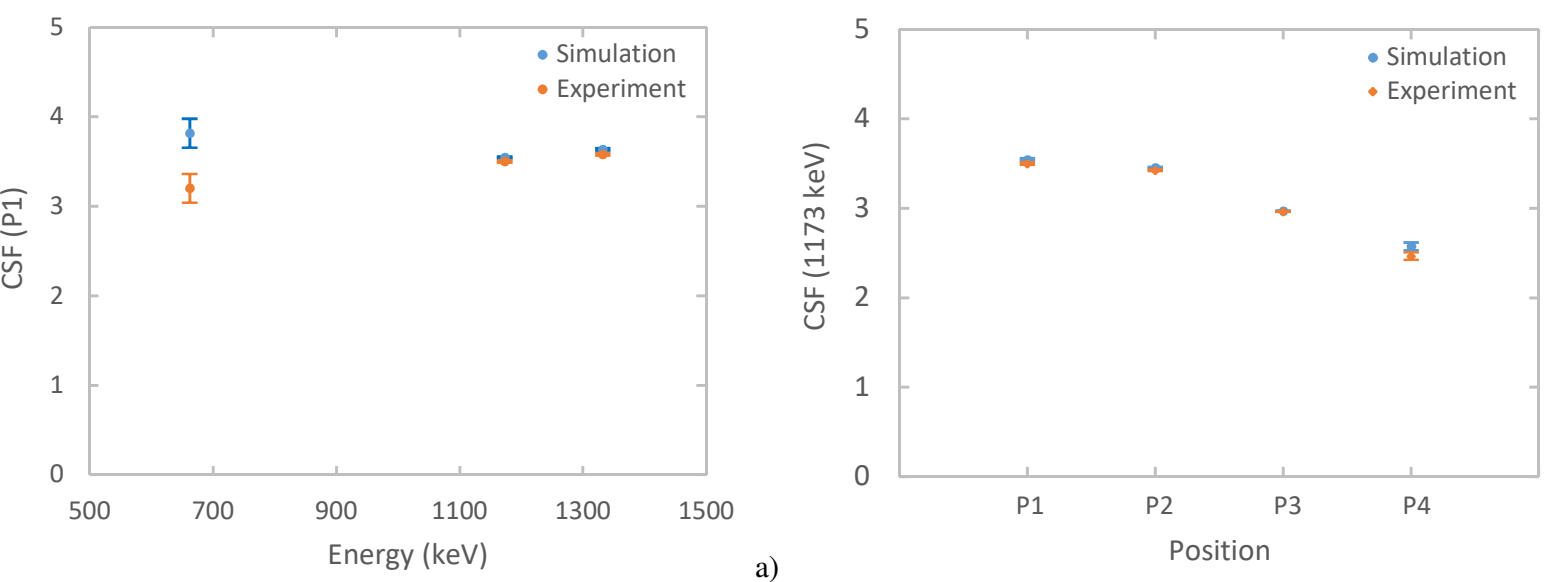

b)

Fig. 6a) Simulated and measured $C S F_{P / C}$ at $\mathrm{P} 1$ as a function on energy, and b) Simulated and measured $C S F_{P / C}$ at $1173.2 \mathrm{keV}$ as a function of position.

The comparison between $C S F_{\text {exp }}$ and $C S F_{\text {sim }}$ indicates that suppression performance are lower in experiments than in simulations. This result was expected on ground of the argument made in the end of Section III. Nevertheless, experimental and simulated $C S F_{P / C}$ agree within an absolute relative error below $5 \%$ in the case of Co- 60 , and below $16 \%$ with Cs-137, so that the model described in Section III accounts reliably for the spectrometric performance of the system. As we are interested in the order of magnitude of the detection limits achievable with an HPGE/BGO CSS, we will therefore base any optimization study of the said system on the simulation based factor of merit $C S F_{s i m}$ without additional experimental correction.

\section{Optimization study of HPGe/BGO CSS}

Geometry is key to maximize the spectrometric performance of a CSS after selecting the natures of primary spectrometer and guard detector. Monte Carlo simulations (MCNP6.1) were thus used to optimize the design of the HPGe/BGO CSS in view of the detection of low levels of uranium activity in large waste containers. The aim of the study is to find the BGO thickness and layout that maximize $C S F_{P / C}$ with minimal degradation of FEP. As we shall discuss with details in paragraph VI.B, the CSS we are designing will be used to investigate the content of waste drums placed in front of the spectrometer (with minimum attenuation in-between), in presence of a- $4 \pi$ environmental background (hence the need for a maximum Compton suppression over the widest angular range surrounding the HPGe diode).

\section{A. Thickness between the inner and outer radii of $B G O$}

The first parameter we varied was the internal radius of the BGO annulus surrounding the HPGe diode. The Compton Suppression Factor was estimated, for the one ray of Cs-137 and both rays of Co-60, as the BGO radial thickness varies from 10 to $70 \mathrm{~mm}$. Such a study should be carried out for a source position corresponding to the target material in a characterization analysis, i.e. position P4 in Fig. 3a). The results of the simulation study are plotted in 


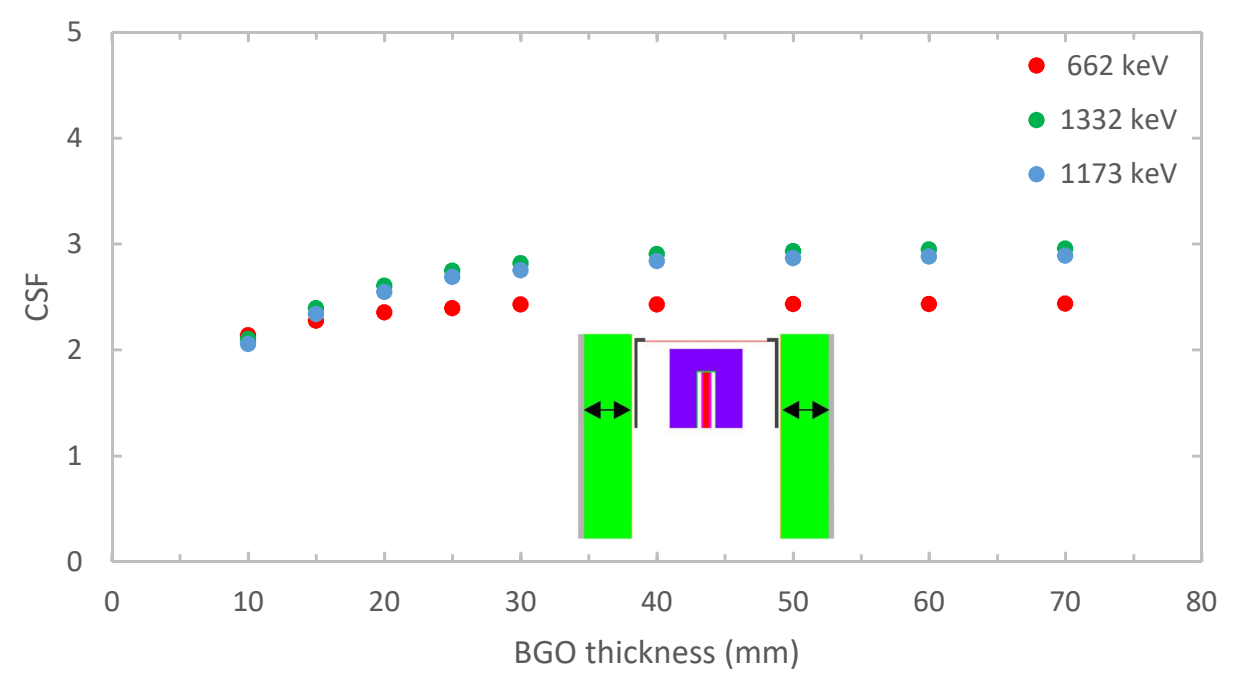

Fig. 7. Evolution of $C S F_{P / C}$ as a function of BGO thickness (Cs-137 and Co-60) at position P4.

All factors of merit $C S F_{P / C}$ increase as a function of BGO thickness. Moreover, the distributions show a convergent trend, with a slope change between 25 and $30 \mathrm{~mm}$ for Co-60 (slope falling from $0.028 \mathrm{~mm}^{-1}$ to $0.012 \mathrm{~mm}^{-1}$ ), and between 15 and $20 \mathrm{~mm}$ for Cs-137 (slope falling from $0.014 \mathrm{~mm}^{-1}$ to $0.008 \mathrm{~mm}^{-1}$ ). As the range of a few hundred of $\mathrm{keV}$ up to $1.5 \mathrm{MeV}$ is representative of the environmental background we are expecting in the context of waste characterisation, we may therefore assume the maximum 30-mm, BGO thickness of the abovementioned values to ensure the convergence of the Compton Suppression Factors underlying the measurement. Additionally, if we consider the value for a 70-mm BGO thickness to be representative of $C S F_{P / C}$ at convergence, the value of Compton Suppression Factor at $30 \mathrm{~mm}$ represents about $95 \%$ of this convergence value for both rays of Co-60, and $99.5 \%$ for Cs-137. The retained thickness thus represents an acceptable compromise with respect to manufacturing costs and transportability on the one hand, and fraction of the maximum Compton suppression achievable on the other hand.

\section{B. Thickness of BGO entrance window}

The PS, in the configurations of paragraph III.D and V.A, is left bare of any BGO entrance window. We hereby model an additional GD coverture topping the entrance window of the HPGe diode, as depicted in Fig. 8. The axial BGO thickness was varied between 5 and $50 \mathrm{~mm}$, and values for $C S F_{P / C}$ with Cs- 137 and Co-60 were estimated. The results of this numerical study are plotted in Fig. 8 as a function of BGO entrance window thickness.

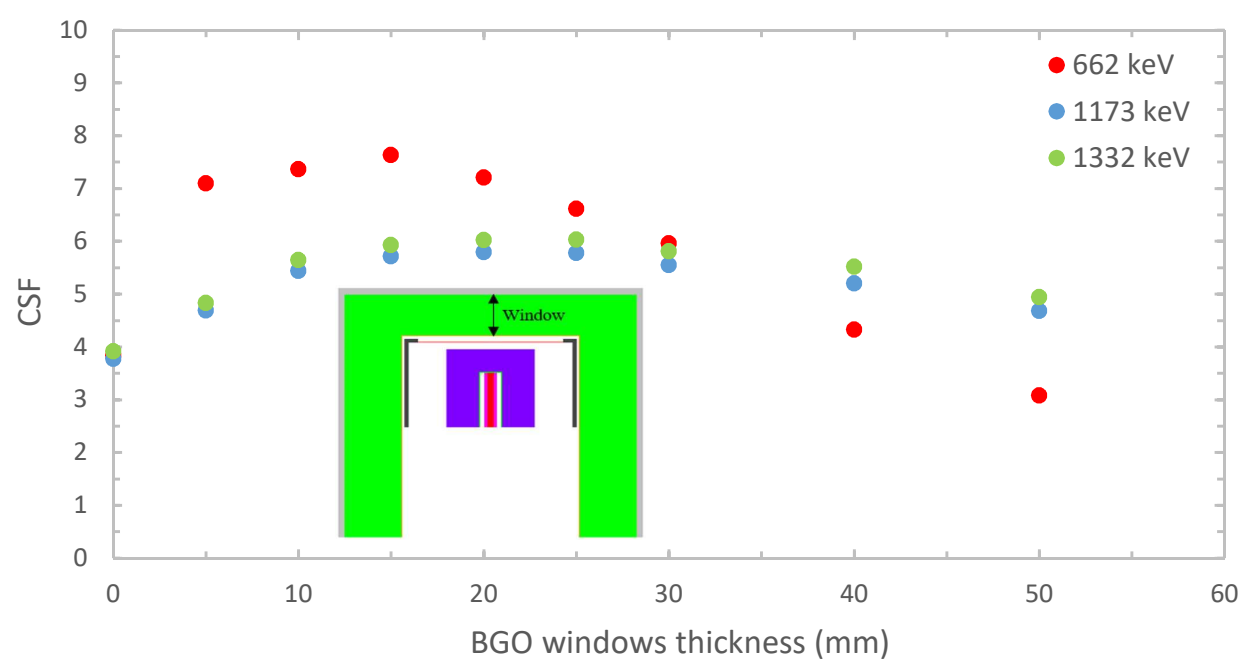

BGO windows thickness $(\mathrm{mm})$

Fig. 8. Evolution of $C S F_{P / C}$ as a function of BGO entrance window thickness (Cs-137 and Co-60) at position P4. 
We obtain the same type of distributions as in paragraph V.A. The characteristics for the two high-energy of Co-60 列 with a 15-mm GBO cap, before dropping quasi-linearly with a slope of about $8.5 \%$ by additional $5 \mathrm{~cm}$. Based on the reunion of these observations, we retained a 15-mm BGO top for our optimized design.

\section{Radius of the BGO collimator hole}

The abrupt decrease of $C S F_{P / C}$, illustrated in the case of Cs-137 (661.7 keV), for BGO entrance windows thicker 491 study therefore involves the scaling of a collimator hole, i.e. an aperture of the BGO coverture. The collimator radius, 492 labelled $r$, is varied between 2.5 and $20 \mathrm{~mm}$ (which corresponds approximately to the diameter of the HPGe diode) as 493 illustrated in Fig. 8. Once the expected signal and background are defined and quantified, the design of in CSS system 494 relies on compromise between the suppression of Compton continuum, generated by high-energy rays, and the 495 maximization of FEP (labelled $P$ ) for the signal of interest, especially critical in the lower-energy region. The 496 evolutions of $C S F_{P / C}$ and $P$ are superimposed in Fig. 9 for collimator radii between 0 and 20 mm and the rays of Cs497137 and Co-60.

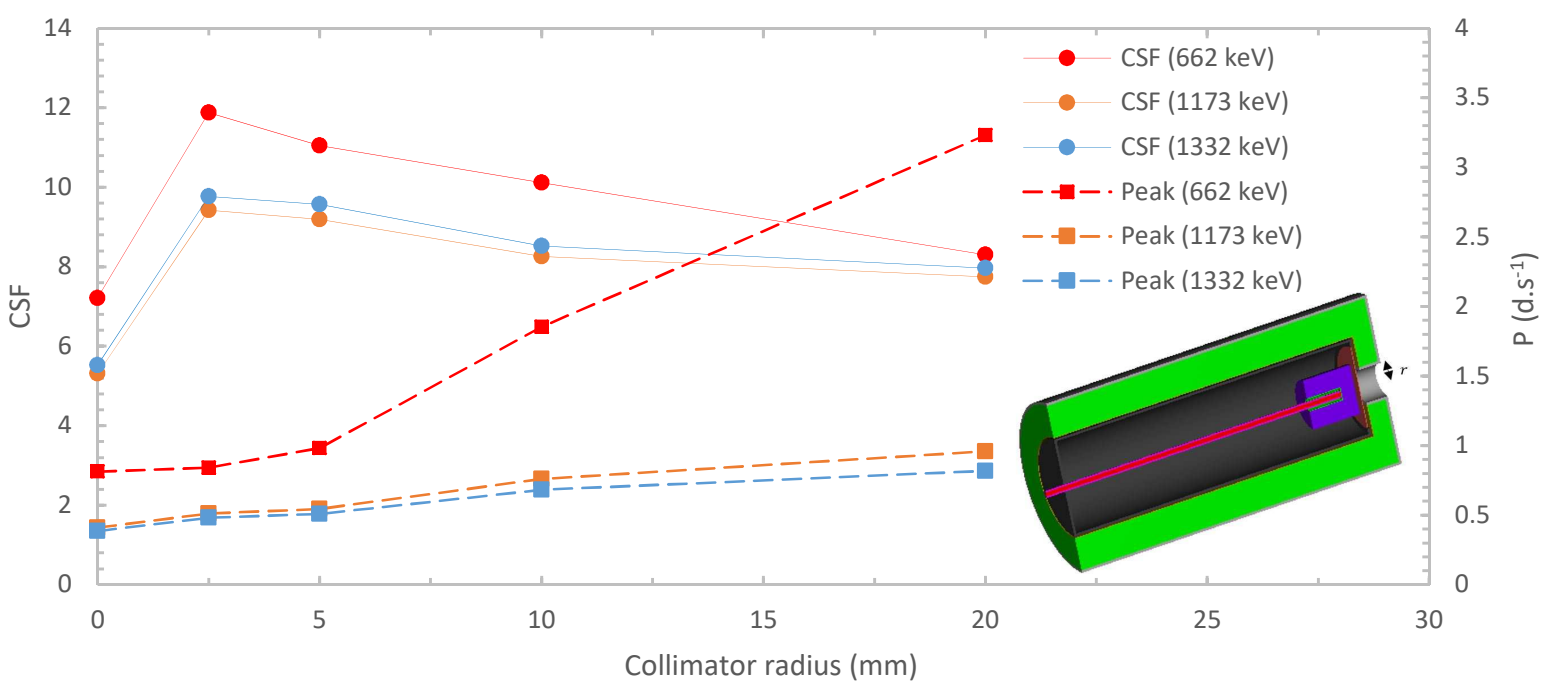

Fig. 9. Evolution of $\operatorname{CSF}_{P / C}$ (line-circle) and $P$ (dotted line-square) as a function of BGO collimator radius (Cs-137, Co-60) at position $\mathrm{P} 4$.

First, we note that the highest values of $C S F_{P / C}$, ranging from 9 (Co-60) to 12 (Cs-137), are reached with a 2.5mm-radius collimator. However, the values of $P$ remain quasi-constant when the radius increases from 0 to $2.5 \mathrm{~mm}$. On the contrary, $P$ increases by a factor of 4 with a $20 \mathrm{~mm}$ radius compared to $2.5 \mathrm{~mm}$, with limited degradation of $C S F_{P / C}$ (8 with $20 \mathrm{~mm}$ against 12 with $2.5 \mathrm{~mm}$ ). A collimator radius of $20 \mathrm{~mm}$ therefore seems to offer an acceptable compromise with respect to signal-to-background ratio.

\section{Estimated performance of CSS final design}

In order to illustrate the optimization procedure detailed in this section, we present the simulated spectra obtained the five following configurations:

- $\quad \mathrm{C}_{1}: 30$-mm-thick BGO cylinder, without entrance window nor anticoincidence filtering;

- $\quad \mathrm{C}_{2}: 30$-mm-thick BGO cylinder, without entrance window, with anticoincidence filtering;

- $\quad \mathrm{C}_{3}: 30$-mm-thick BGO cylinder, 15-mm entrance window, no collimator, with anti-coincidence filtering;

- $\quad \mathrm{C}_{4}$ : 30-mm-thick BGO cylinder, 15-mm entrance window, 40-mm(Ø) collimator, with anticoincidence filtering.

The obtained spectra are reproduced in Fig. 9 for a Cs-137 simulated emitter at P4 (10 cm from the PS window). 


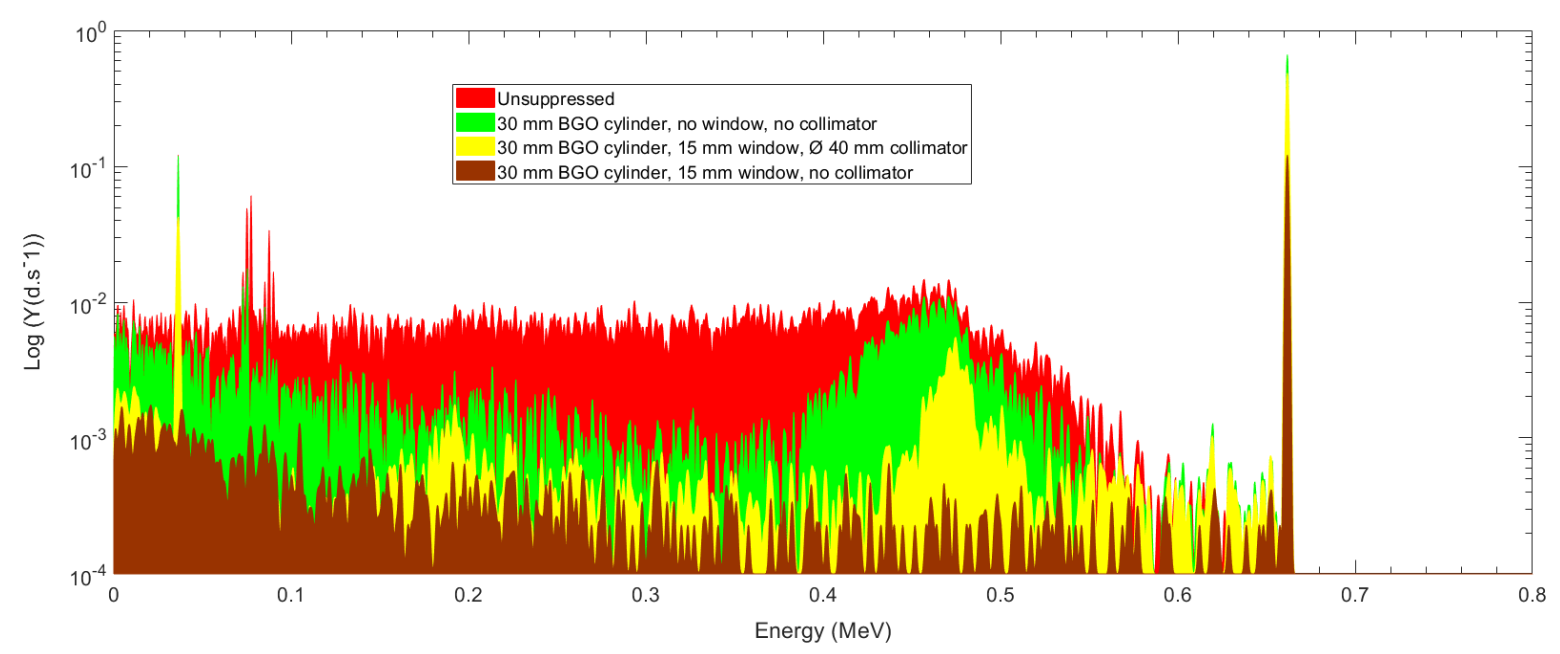

Fig. 10. Superimposition of HPGe spectra without BGO and with various BGO shield configurations (Cs-137)

In configuration $\mathrm{C}_{3}$ (brown spectrum), we verify that adding a 15 -mm window without any collimator hole reduces considerably the Compton background. Such an effect is due to the $4 \pi$ coverage of the HPGe solid angle by the BGO guard detector. A noticeable consequence of this coverage is the quasi-complete suppression of the Compton edge, with high-energy electron depositions confined to the scintillator, and residual photons scattering back to the source. We also highlight the suppression of the $31-37 \mathrm{keV} \mathrm{X}$ rays from internal conversions, due to the absence of an aperture in configuration $\mathrm{C}_{3}$. Eventually, such a design degrades the FEP signal-of-interest at $661.7 \mathrm{keV}$, reducing it by about half an order of magnitude.

At the same time, we observe that, by adding a 40-mm(Ø) collimator (configuration $\mathrm{C}_{4}$, yellow spectrum), a oneorder-of magnitude reduction of Compton background is still ensured when compared to $\mathrm{C}_{1}$ (red curve), while conserving about $75 \%$ of the FEP area in $\mathrm{C}_{1}$. The geometrical configuration detailed in paragraph IV.C and illustrated in curve $\mathrm{C}_{4}$ will therefore be used in the next section to derive the expected detection limits of natural uranium under a representative radiological background.

\section{Detection limits for natural uranium in waste containers with HPGe/BGO CSS}

\section{A. Signal, background, and detection limit}

The uranium to be detected in waste containers is supposed to be descripted by the said "natural" isotopic fractions of U-234, U-235, and U-238. In such conditions, U-238 represents an isotopic fraction of $99.2745 \%$, and accounts for $48.07 \%$ of total uranium activity. It is thus the gamma-ray signature of the decay of this isotope that is preferentially used to detect and quantity uranium under these hypotheses [37]. This gamma-ray signature appears as a result of nuclear decays and de-excitations along the radioactive chain of U-238, whose first features are

\section{Decay chain of U-238}

$$
\begin{gathered}
{ }_{92}^{238} \mathrm{U} \rightarrow{ }_{90}^{234} \mathrm{Th}+{ }_{2}^{4} \alpha(4.468 \mathrm{MeV}) \\
{ }_{90}^{234} \mathrm{Th} \rightarrow{ }_{91}^{234} \mathrm{~Pa}^{2} \mathrm{~m}+{ }_{-1}^{0} \beta^{-}(272 \mathrm{keV})+{ }_{0}^{0} \overline{v_{e}} \\
\rightarrow{ }_{99}^{234} \mathrm{~Pa}+{ }_{-1}^{0} \beta^{-}(272 \mathrm{keV})+{ }_{0}^{0} \bar{v}_{e}+\gamma \\
{ }_{91}^{234} \mathrm{~Pa}^{0} \mathrm{~m} \rightarrow{ }_{92}^{234} \mathrm{U}+{ }_{-1}^{0} \beta^{-}(2.195 \mathrm{MeV})+{ }_{0}^{0} \bar{v}_{e}+\gamma \ldots
\end{gathered}
$$

The emission yields for the main gamma rays are tabulated [38]. By taking into the gamma-ray emission intensities and the amplitude of background in the associated spectral regions, the isotopic detection limit of U-238 is the one of the 1001.026-keV line [39]. The said gamma ray follows the de-excitation of U-234*, which itself comes from the beta-decay of Pa-234m. The emission intensity reads $I_{\gamma}(1001 \mathrm{keV})=8.47 \cdot 10^{-3} \gamma \cdot \mathrm{dis}^{-1}$ of Pa-234m. As far as the U-238 chain is concerned, a 99.9-\% equilibrium between the activities of U-238 and Pa-234m is reached with approximately eight months. We consider that such an equilibrium exists. It is then possible to deduce the isotopic activity of U-238, and therefore the elementary activity of uranium, using the emission yield $\eta_{\gamma}(1001 \mathrm{keV})=3.88$. $10^{-3} \gamma . \mathrm{Bq}^{-1}$ of the identified line per Becquerel of natural uranium. 
$565 F W H M(1001 \mathrm{keV})=2.6 \mathrm{keV}$. It follow that the region of interest $R O I$ over which we aim at detecting the presence 566 of uranium is given by:

$$
R O I(1001 \mathrm{keV})=[1001 \mathrm{keV} \pm 1.25 \cdot F W H M(1001 \mathrm{keV})]=[998.3 \mathrm{keV} ; 1003.6 \mathrm{keV}]
$$

The characterization of waste containers is conducted under a radiological background about which we are also to make some assumptions. We thus suppose that the spectrometry chain is installed inside a close, laboratory-type environment. In such an environment, the background gamma-ray emission is dominated by K-40, and the decay products of the radioactive chains of U-238 and Th-232 [39,40]. The main characteristic lines are listed in Table VI, where we mention the nuclide whose de-excitation they indicate and the unstable parent of the said nuclide. In order to simulate the interaction of a typical background with our HPGe/BGO CSS, it is additionally necessary to define the relative contributions of the gamma rays. To this end, we make us of the source term estimated by Medhat et al. [41] in a similar environment, which we reproduced in Table VI. This source term includes the main lines of Ra-226, Bi$214, \mathrm{~Pb}-214$ and 212, Tl-208 and K-40. The contribution from other radionuclides such as $\mathrm{Pb}-210$ were left out due to low emission probability $(4.2 \%)$ and low energy range $(46 \mathrm{keV})$. The normalized source spectrum is finally multiplied by a constant so that the resulting energy deposition spectrum, as obtained when the HPGe is simulated alone, has the same counting statistics (in s${ }^{-1}$ ) as a background spectrum acquired inside the test room of Section IV.

Table VI. Gamma rays and relative contributions associated to the simulation of background [41].

\begin{tabular}{cccc}
\hline Energy $(\mathrm{keV})$ & Radionuclide & Decay product & Relative contribution $(\%)$ \\
\hline 186.0 & Ra-226 & Rn-222* & 1.0 \\
609.3 & Bi-214 & Po-214* & 14.5 \\
768.4 & Bi-214 & Po-214* & 1.6 \\
1120.3 & Bi-214 & Po-214* $(\gamma)$ & 4.7 \\
1764.0 & Bi-214 & Po-214* $(\gamma)$ & 5.0 \\
295.2 & Bi-214 & Po-214* $(\gamma)$ & 6.0 \\
351.9 & $\mathrm{~Pb}-214$ & Bi-214* $(\gamma)$ & 11.7 \\
338.0 & Ac-228 & Th-228* $(\gamma)$ & 3.6 \\
911.1 & Ac-228 & Th-228* $(\gamma)$ & 8.7 \\
969.1 & Ac-228 & Th-228* $(\gamma)$ & 5.2 \\
238.6 & $\mathrm{~Pb}-212$ & $\mathrm{Bi}-212^{*}(\gamma)$ & 14.0 \\
583.1 & $\mathrm{Tl}-208$ & $\mathrm{~Pb}-208^{*}(\gamma)$ & 9.4 \\
2614.5 & $\mathrm{Tl}-208$ & $\mathrm{~Pb}-208^{*}(\gamma)$ & 11.2 \\
1460.8 & $\mathrm{Kr}-40 *(\gamma)$ & 3.4 \\
\hline
\end{tabular}

The capacity of a spectrometry chain to identify and deconvolve the FEP of the isotopic signal of interest is determined by the expected count rate inside the ROI in the absence of uranium. If we label $B\left(E_{j}\right)$ the simulated spectral response of the primary spectrometer to the background gamma-ray emission, this deposition yield, which can be seen as a baseline, reads

$$
B(1001 \mathrm{keV})=\sum_{E_{j} \in R O I(1001 \mathrm{keV})} B\left(E_{j}\right)
$$

The detection limit of the gamma-ray signature is defined with respect to two error risks:

- $\quad$ the type I risk, corresponding to false detections, labeled $\alpha$ and expressed in $\%$;

- $\quad$ the type II risk, corresponding to false non-detections, labeled $\beta$ and also expressed in $\%$.

It is customary, in the absence of any ad hoc specification, to set $\alpha=\beta=2.5 \%$. The detection limit at the end of a counting experiment of duration $t$, labeled $D L_{2.5 \%}$ and expressed in counts (hereby equivalent to simulated energy depositions from the conclusions of paragraphs IV.2 and IV.3), is then given by [42]:

$$
D L_{2.5 \%}=1.96^{2}+3.92 \sqrt{2 \cdot B(1001 \mathrm{keV}) \cdot t}
$$

This detection limit is converted into a minimal detectable mass activity $M D M A_{2,5 \%}$ of uranium using the equation

$$
M D M A_{2.5 \%}=\frac{D L_{2.5 \%}}{T(1001 \mathrm{keV}) \cdot \eta_{\gamma}(1001 \mathrm{keV}) \cdot m \cdot t}
$$


where $T(1001 \mathrm{keV})$ is, as in the previous sections, the full energy absorption efficiency of the 1001-keV ray and $m$ 607 the mass of the characterized object, in this case a waste container.

608 By combining Eq. (13) and (14), we see that, the constant left aside in the detection limit, we have the following 609 proportional relationship:

$$
M D M A_{2.5 \%} \propto \frac{\sqrt{B(1001 \mathrm{keV})}}{T(1001 \mathrm{keV}) \sqrt{t}}
$$

612

This expression sums up the trade-off to reach so that the HPGe/BGO CSS proves itself to be a relevant solution to the problem of low-activity detection: ensuring a maximum reduction of diffusion background $B$ while keeping the degradation of the FEP efficiency $T$, all with the purpose of lowering the minimal detectable mass activity for a constant acquisition time $t$.

\section{B. Simulation of the measurement}

The simulation study is carried out by means of the Particle TRAck post-processing code described throughout Section III, and experimentally validated in Section IV.

The standard container to be characterized is modelled as a perfect cylinder, homogenously filled with a mixture of lime and sorbent described by the chemical formula $\mathrm{Ca}(\mathrm{OH})_{2}$. The main parameters used in the model include a net mass of $78 \mathrm{~kg}$, a filling height of $86 \mathrm{~cm}$, an external radius of $28.65 \mathrm{~cm}$, a radial thickness of $0.15 \mathrm{~cm}$, and a density of $7.8 \mathrm{~g} . \mathrm{cm}^{-3}$.

The HPGe diode, with and without the BGO active shielding (as optimized in Section V) is set horizontally so that:

- the center of mass of the crystal is at the same height as the center of mass of the filled part of the container;

- the crystal entrance window is at $1 \mathrm{~m}$ from the external envelope of the container.

Regarding the simulation of gamma-ray background, we consider that a container without uranium induces no alteration of the spectrum, nor any increase of count rate $B$. We therefore generate a source term corresponding to the data in Table VI, centred on the HPGe crystal, homogenous and isotropic inside the measurement environment. As far as the signal from natural uranium is concerned, we generate a volumic source term, also homogenous and isotropic, of a photon nature and 1001-keV energy, inside the filled part of the modelled container

The useful estimates at the output of the simulation scheme are:

- the background $B_{H P G e}(1001 \mathrm{keV})$ inside the $R O I$ when the HPGe is used without active shielding;

- the FEP efficiency $T_{H P G e}(1001 \mathrm{keV})$ inside the ROI when the HPGe is used without active shielding;

- the background $B_{C S S}(1001 \mathrm{keV})$ inside the ROI when the HPGe is used as part of the HPGe/BGO CSS with anticoincidence filtering;

- the FEP efficiency $T_{H P G e}(1001 \mathrm{keV})$ inside the ROI when the HPGe is used as part of the HPGe/BGO CSS with anticoincidence filtering.

These results are presented and discussed in the next paragraph.

\section{Results}

We present in Fig. 11 the superimposition of estimated spectral responses $B_{H P G e}\left(E_{j}\right)$ and $B_{C S S}\left(E_{j}\right)$, for $E_{j}$ lying between 0 and $3 \mathrm{MeV}$, in presence of the simulated gamma-ray background. The FEP associated to some of the prominent lines in Table VI are also indicate in the figure. 


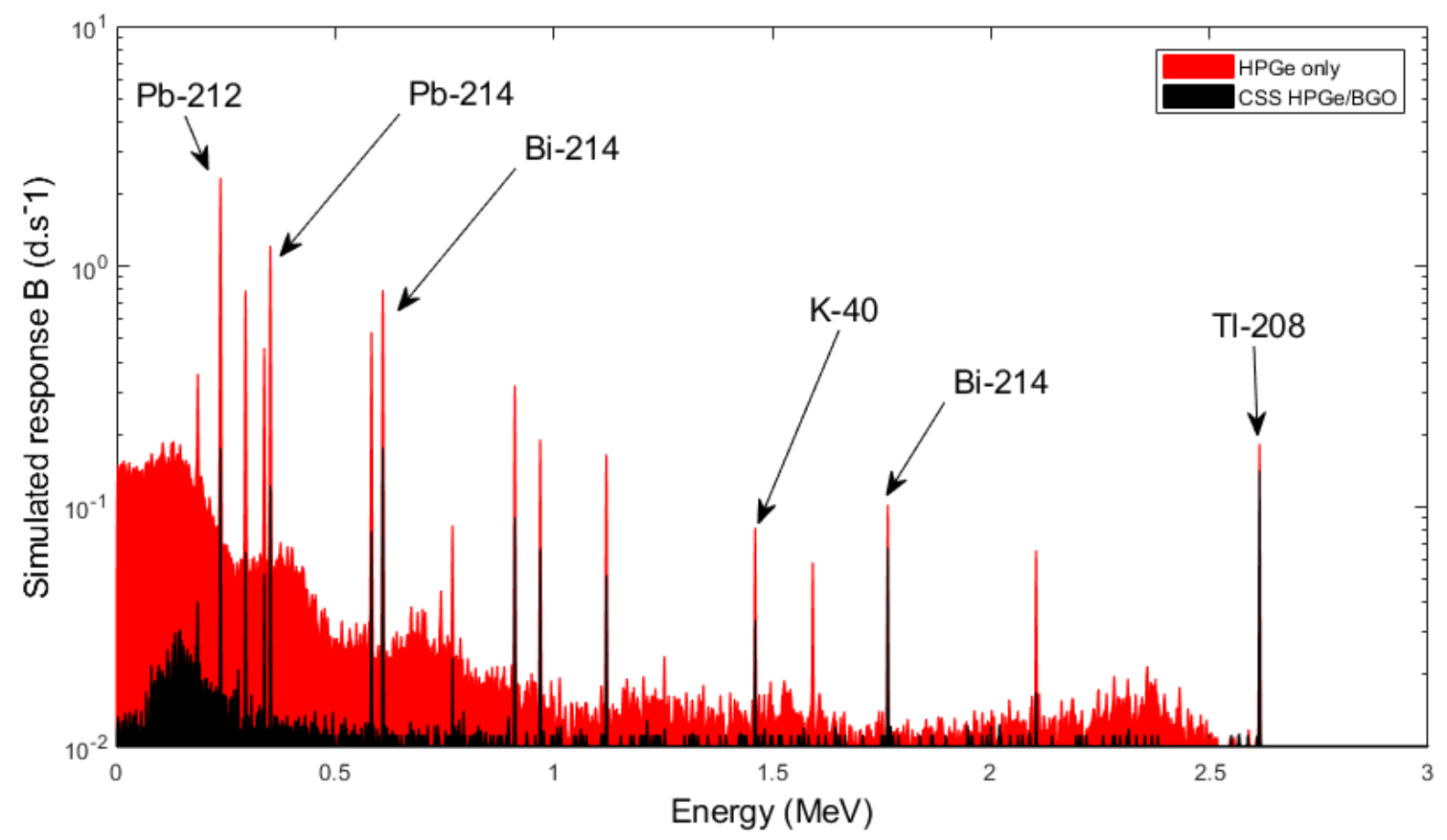

Fig. 11. Simulated spectral responses $B_{H P G e}\left(E_{j}\right)$ and $B_{C S S}\left(E_{j}\right)$.

The distributions in Fig. 11 show a drastic reduction of the FEP as well as of the Compton diffusion continuum, around one order of magnitude above $500 \mathrm{keV}$, and up to almost one order and a half below $50 \mathrm{keV}$. In particular, over the $R O I$ defined in view of the identification of uranium, we obtain by simulation a continuum suppression

$$
\frac{B_{C S S}(1001 \mathrm{keV})}{B_{\text {HPGe }}(1001 \mathrm{keV})} \sim 9.85
$$

Meanwhile, we observe a reduction of the net area under the FEP at $1001 \mathrm{keV}$

$$
\frac{T_{C S S}(1001 \mathrm{keV})}{T_{H P G e}(1001 \mathrm{keV})} \sim 0.77
$$

It follows by Eq. (15) that we are expecting a lowering of the minimal detectable mass activity by a factor of 2.5 approximately when implementing the CSS we have designed. Indeed, numerical applications from Eq. (13) and (14), show that the minimal detectable mass activity goes from $M D M A_{H P G e, 2,5 \%}=8.5 \pm 1.4 \mathrm{~Bq} \cdot \mathrm{g}^{-1} \cdot \mathrm{h}^{-1}$ without active shielding down to $M D M A_{C S S, 2,5 \%}=3.5 \pm 0.7 \mathrm{~Bq} \cdot \mathrm{g}^{-1} \cdot \mathrm{h}^{-1}$ with the HPGe/BGO CSS.

\section{Conclusion}

The aim of the study was to assess the opportunity of a Compton suppression system (CSS) to detect low activities of uranium inside large waste containers. The proposed CSS is based on an HPGe diode as a primary spectrometer (PS). Among several scintillator candidates, we justified the choice of a BGO crystal as guard detector (GD), mainly due to the high linear attenuation coefficient of this material. We then detailed the conception study of an HPGe/BGO CSS based MCNP6.1 Particle TRAck files and a dedicated reconstruction code. The numerical scheme, which simulated the filtering of multiple-diffusion events inside both the PS and the GD, was validated though a series of measurements. Taking advantage of this validation, we carried out an optimization study of the BGO active shield with respect to Compton suppression factor and FEP efficiency. In the final step of this work, we estimated by simulation the reduction of minimal detectable mass activity of uranium inside a waste container, as ensured by an HPGe/BGO CSS in presence of environmental gamma-ray background.

The results of the study show that a reduction of minimal detectable mass activity by a factor close to 2.5 is expected. This means that the detection, with $97.5-\%$ chance, of a natural uranium mass activity of $1 \mathrm{~Bq}^{-\mathrm{g}^{-1}}$ would require a six times lower acquisition time with the CSS hereby described than with the HPGe diode only, one meter from the container. This estimated justifies the relevance of an active shielding technological solution to the problem of the detection of low-activity radiological waste in containers. 
If a gain by a factor of six on acquisition duration is valuable result, one should highlight that the integration time required for low mass activity detection remains high: about twelve hours and a half for $1 \mathrm{~Bq}^{-1} \mathrm{~g}^{-1}$, with alpha and beta risks of $2.5 \%$. In order to lower again detection limits, and therefore the duration of the characterizing procedure, we will focus our next work on the filtering of additional components in the diffusion continuum. The main strategies to achieve such a filtering will involve the exploitation of the spectrometric information available at the level of GD itself, as well as the voxelization of the said detector [43].

\section{Acknowledgements}

The authors would like to thank M. Venaruzzo and his team (CAEN) for their help during this work, as well as Z. Mekhalfa and R. Abou Khalil (ORANO DS) for funding this research.

\section{References}

[1] G. R. Gilmore, Practical Gamma-ray Spectrometry, $2^{\text {nd }}$ Edition, John Wiley \& Sons Ltd, The Atrium, Southern Gate, Chichester, West Sussex, England, pp. 41-47 (2008).

[2] R. J. Cooper, M. Amman, K. Velter, "High resolution gamma-ray spectroscopy at high count rates with a prototype High Purity Germanium”, Nuclear Instruments and Methods in Physics Research A, Vol. 886, pp. 1-6 (2018).

[3] G. S. C. Joela, S. Penabeic , M. M. Ndontchueng, G. Chenea, E. J. N. Mekontso, A. N. Ebongue, M. Ousmanoub , S. David, "Precision measurement of radioactivity in gamma-rays spectrometry using two HPGe detectors (BEGe6530 and GC0818-7600SL models) comparison techniques: Application to the soil measurement", MethodsX, Vol. 4, pp. $42-54$ (2017).

[4] E. Rohée, R. Coulon, F. Carrel, T. Dautremer, E. Barat, T. Montagu, S. Normand, C. Jammes, "Benchmark of the non-parametric Bayesian deconvolution method implemented in the SINBAD code for X/Y rays spectra processing", Nuclear Instruments and Methods in Physics Research A, Vol. 836, pp. 91-97 (2016).

[5] M. L. Simpson, T. H. Becker, R. D. Bingham, R. C. Trammell, "An Ultra-High-Throughput, High-Resolution, Gamma-Ray Spectroscopy System”, IEEE Transactions on Nuclear Science, Vol. 38, № 2, pp. 89-96 (1991).

[6] E. Barat, T. Dautremer, T. Montagu, J.-C. Trama, “A bimodal Kalman smoother for nuclear spectrometry” Nuclear Instruments and Methods in Physics Research A, Vol. 567, pp. 350-352 (2006).

[7] S. Shun, X. Cai, Z. Wu, Y. Xie, B. Yu, Z. Wang, J. Fang, X. Sun, L. Sun, Y. Liu, L. Gao, X. Zhang, H. Zhao, L. Zhou, J. Lv, T. Hu, "Simulation of background reduction and Compton depression in low-background HPGe spectrometer at a surface laboratory", Chinese Physics C, Vol. 39, N 8, 086002, 1-7 (2015).

[8] S. M. Judge, P. H. Regan, “ Radionuclide metrology research for nuclear site decommissioning”, Radiation Physics and Chemistry, Vol. 140, pp.463-465 (2017).

[9] C. Chung, L. Yuan, "Performance of a HPGe-NaI (TI) Compton Suppression Spectrometer in high-level radioenvironmental studies", Nuclear Instruments and Methods in Physics Research A, Vol. 243, pp.102-110 (1986).

[10] R. Coulon et al, "Delayed gamma power measurement for sodium-cooled fast reactors", Nuclear Engineering and Design, Vol. 241, pp. 339-348 (2011).

[11] G. F. Knoll, "Radiation Detection and Measurement”, 4th ed, Wiley, pp 779 (2010).

[12] L. E. Smith, Z. He, D. K. Wehe, G. F. Knoll, "Pulse Processing Electronics with Anticoincident Circuitry for a Multi-Channel Portable Gamma Camera”, IEEE Transactions on Nuclear Science, Vol. 45, N³, pp. 963-969 (1998).

[13] R. Britton, J. L. Brunett, A. V. Davies, P. H. Regan, "Monte-Carlo optimisation of a Compton suppression system for use with a broad-energy HPGe detector" Nuclear Instruments and Methods in Physics Research A, Vol. 762 , pp. $42-53$ (2014). 
[14] Q. Zou, J. J. Zhu, Z. An, Y. P. Zhang, "Monte Carlo optimization of a Compton suppression system for gammaray diagnosis of combustion plasma" Nuclear Instruments and Methods in Physics Research A, Vol. 879, pp. 57-63 (2018).

[15] IEEE Standard Test Procedures for Germanium Gamma-Ray Detectors, American National Standard/IEEE, Std 325-1996 (1996).

[16] D. Massé, A. Adam, J. Laurec, "A Ge-NaI(Tl) spectrometer with Compton suppression and gamma coincidence counting. Application to ${ }^{189}$ Ir and ${ }^{101}$ Rh activity", Nuclear Instruments and Methods in Physics Research A, Vol. 309, pp. 2271-235 (1991).

[17] C. Chung, L.-J. Yuan, "Performance of a HPGe-NaI(Tl) Compton suppression spectrometer in high-level radio environmental studies", Nuclear Instruments and Methods, Vol 243, pp. 102-110 (1986).

[18] K. Fukuda, J. ohkuka, "Design of a Ge-GBO Compton-suppression spectrometer and its application in photon activation analysis", Technical report ISIR Osaka University.

[19] S. L. Tabor, "BGO suppressed gamma detector arrays", Nuclear Instruments and Methods B, Vol 24-25, pp. 1031-1034 (1987).

[20] H. J. M. Aarts, G.A.P. Engelbertink, G.L. Van Der Poel, D.E.C. Scherpenzeel, H.F.R. Arciszewski, "Improvement of the performance of a Compton suppression spectrometer by minimizing the dead layer of the central Ge detector", Nuclear Instruments and Methods, Vol 172, pp. 439-446 (1980).

[21] H.J.M. Aarts, C.J. Van Der Poel, D.E.C. Scherpenzeel, H.F.R. Arciszewski, G.A.P. Engelbertink, “A Compton suppression spectrometer for gamma-gamma coincidence measurements: large solid angle and excellent suppression", Nuclear Instruments and Methods, Vol 177, pp. 417-425 (1980).

[22] P.J. Nolan, D.W. Gifford, P.J. Twin, “The performance of a bismuth germanate escape suppressed spectrometer”, Nuclear Instruments and Methods A, Vol 236, pp. 95-99 (1985).

[23] D. Massé, “A Ge-NaI (Tl) spectrometer with Compton suppression and gamma coincidence counting”, Technical report CEA-R-5539 (1990).

[24] K. Iniewski, "Semiconductor Radiation Detection Systems”, CRC Press, pp 368 (2018).

[25] Germanium Detectors User's Manual 9231358B, Canberra, Copyright (2003).

[26] R. Coulon, J. Dumazert, S. Garti, "Procédé de caractérisation d'un spectromètre, produit-programme d'ordinateur et calculateur associés", French Atomic Energy Commission, Patent deposited on 17/12/2018 under n FR1873097 (2018).

[27] Moritz Geometry Tool, Version 1.23, An Interactive Geometry Editor/Viewer for MCNP, White Rock Science, Los Alamos, New Mexico (2012).

[28] Goorley, John T, MCNP6 User's Manual Version 1.0, Los Alamos National Lab. (LANL), Los Alamos, NM (United States), , LA-UR-13-22934 (2013).

[29] J. Allison et al., "Recent developments in GEANT4", Nuclear Instruments and Methods in Physics Research A, Vol. 835, pp. 186-225 (2016).

[30] E. E. Zadeh, S. A. H. Feghhi, E. Bayat, G. H. Roshani, "Gaussian Energy Broadening Function of an HPGe Detector in the Range of $40 \mathrm{keV}$ to $1.46 \mathrm{MeV}$ ", Journal of Experimental Physics, Vol 2014, Article ID 623683, pp. 15 (2014).

[31] G. R. Gilmore, Practical Gamma-ray Spectrometry, $2^{\text {nd }}$ Edition, John Wiley \& Sons Ltd, The Atrium, Southern Gate, Chichester, West Sussex, England, pp. 131-141 (2008).

[32] MATLAB and Statistics Toolbox Release 2017b, The MathWorks, Inc., Natick, Massachusetts, United States (2017). 
[33] Periodic Table of Elements and $X$-ray Energies www.bruker.com/hhxrf

818 [39] M. Tsutsumi, T. Oishi, N. Kinouchi, R. Sakamoto, M. Yoshida, "Simulation of the background for gamma 819 detection system in the indoor environment of concrete buidings", Journal of Nuclear Science and Technology, Vol. $82038, \mathrm{~N}^{\circ} 12$, pp. 1109-1114 (2001).

[34] https://scionix.nl/wp-content/uploads/2017/07/Compton-suppression-shields.pdf

[35] https://www.caensys.com/mc2analyzer/

[36] G. F. Knoll, Radiation Detection and Measurement, $4^{\text {th }}$ Edition, Wiley, pp 90-92 (2010).

[37] M. Hult, E. Andreotti, R. González de Orduña, S. Pommé, Y. Yeltepe, "Quantification of uranium-238 in environmental samples using gamma-ray spectrometry", EPJ Web of Conferences, Vol. 24, 07005 (2012).

[38] M.-M. Bé, V. Chisté, Table de radionucléides, LNE-LNHB/CEA (2007).

[40] T. Marchais, B. Pérot, C. Carasco, P.-G. Allinei, P. Chaussonnet, J.-L. Ma, H. Toubon, "Gamma-ray spectroscopy measurements and simulations for uranium mining", EPJ Web of Conferences, Vol. 170, 05003 (2018).

[41] M. E. Medhat, Y. Wang, "Estimation of background spectrum in a shielded HPGe detector using Monte Carlo simulations", Applied Radiation and Isotopes, Vol. 84, pp. 13-18 (2014).

[42] ISO11929-3, Determination of the detection limit and decision threshold for ionizing radiation (2010).

[43] R. Coulon, J. Dumazert, S. Garti, Q. Lecomte, "Système de spectrométrie, procédé de spectrométrie et produit programme d'ordinateur associés", French Atomic Energy Commission, Patent deposited on 27/09/2018 under $n^{\circ}$ FR1858897 (2018). 